\title{
Author Correction: Building a global alliance of biofoundries
}

\author{
Nathan Hillson (1) 1, Mark Caddick (1) 2, Yizhi Cai (10) ${ }^{3}$, Jose A. Carrasco ${ }^{4}$, Matthew Wook Chang (1) ${ }^{5}$, \\ Natalie C. Curach (1) ${ }^{6}$, David J. Bell (1) ${ }^{7}$, Rosalind Le Feuvre ${ }^{3}$, Douglas C. Friedman (1) ${ }^{8}$, Xiongfei Fu (1) ${ }^{9}$, \\ Nicholas D. Gold ${ }^{10}$, Markus J. Herrgård ${ }^{11}$, Maciej B. Holowko (10 12,13,14, James R. Johnson (10 2, \\ Richard A. Johnson ${ }^{15}$, Jay D. Keasling (1) 1, Richard I. Kitney (1) 7, Akihiko Kondo (1) ${ }^{16}$, Chenli Liu (D) 9 , \\ Vincent J.J. Martin (1) ${ }^{10}$, Filippo Menolascina ${ }^{17}$, Chiaki Ogino (1) ${ }^{16}$, Nicola J. Patron (1) ${ }^{4}$, Marilene Pavan ${ }^{18}$, \\ Chueh Loo Poh ${ }^{5}$, Isak S. Pretorius (10 ${ }^{19}$, Susan J. Rosser ${ }^{17}$, Nigel S. Scrutton ${ }^{3}$, Marko Storch (D) ${ }^{7}$, Hille Tekotte ${ }^{17}$, \\ Evelyn Travnik ${ }^{11}$, Claudia E. Vickers ${ }^{12,13}$, Wen Shan Yew ${ }^{5}$, Yingjin Yuan (1) ${ }^{20}$, Huimin Zhao (1) ${ }^{21}$ \& \\ Paul S. Freemont (iD ${ }^{7}$
}

Correction to: Nature Communications https://doi.org/10.1038/s41467-019-10079-2, published online 9 May 2019.

The original version of this Comment contained errors in the legend of Figure 2, in which the locations of the fifteenth and sixteenth GBA members were incorrectly given as '(15) Australian Genome Foundry, Macquarie University; (16) Australian Foundry for Advanced Biomanufacturing, University of Queensland'. The correct version replaces this with '(15) Australian Foundry for Advanced Biomanufacturing (AusFAB), University of Queensland and (16) Australian Genome Foundry, Macquarie University'. This has been corrected in both the PDF and HTML versions of the Comment.

Published online: 11 July 2019

\footnotetext{
${ }^{1}$ DOE Agile BioFoundry, Emeryville, CA 94608, USA. ${ }^{2}$ GeneMill, Institute of Integrative Biology, University of Liverpool, Liverpool L69 7ZB, UK. ${ }^{3}$ SYNBIOCHEM, Manchester Institute of Biotechnology and School of Chemistry, University of Manchester, Manchester M13 9PL, UK. ${ }^{4}$ Earlham Institute, Norwich Research Park, Norfolk NR4 7UZ, UK. ${ }^{5}$ NUS Synthetic Biology for Clinical and Technological Innovation (SynCTI), Department of Biochemistry, Yong Loo Lin School of Medicine, National University of Singapore, Singapore 117456, Singapore. ${ }^{6}$ Bioplatforms Australia, Research Park Drive, Macquarie University, Macquarie Park, NSW 2109, Australia. ${ }^{7}$ London DNA Foundry, Imperial College Translation \& Innovation Hub, White City Campus, 80 WoodLane, London W12 OBZ, UK. ${ }^{8}$ Engineering Biology Research Consortium (EBRC), Emeryville, CA 94608, USA. ${ }^{9}$ Shenzhen Institute of Synthetic Biology, Shenzhen Institutes of Advanced Technology, Chinese Academy of Sciences, Shenzhen, People's Republic of China. ${ }^{10}$ Centre for Applied Synthetic Biology, Concordia University, Montreal, Montreal, QC H4B 1R6, Canada. ${ }^{11}$ The Novo Nordisk Foundation Center for Biosustainability, Technical University of Denmark, 2800 Kongens Lyngby, Denmark. ${ }^{12}$ CSIRO Synthetic Biology Future Science Platform, Canberra ACT 2601, Australia. ${ }^{13}$ Australian Institute for Bioengineering and Nanotechnology, The University of Queensland, Brisbane, QLD 4072, Australia. ${ }^{14}$ Department of Molecular Sciences, Macquarie University, Macquarie, NSW 2109, Australia. ${ }^{15}$ Global Helix LLC, BioBricks Foundation, and Engineering Biology Research Consortium (EBRC), Emeryville CA 94608, USA. ${ }^{16}$ Graduate School of Science, Technology, and Innovation, Kobe University, Kobe 657-8501, Japan. ${ }^{17}$ UK Centre for Mammalian Synthetic Biology SynthSys, School of Biological Sciences, University of Edinburgh, Edinburgh EH93FF, UK. ${ }^{18}$ DAMP Lab, Biological Design Center, Boston University, Boston, MA 02215, USA. ${ }^{19}$ Macquarie University, North Ryde, NSW 2109, Australia. ${ }^{20}$ Frontier Science Center for Synthetic Biology (MOE),

TianjinUniversity, Tianjin, People's Republic of China. ${ }^{21}$ Illinois Biological Foundry for Advanced Biomanufacturing (iBioFAB), University of Illinois at UrbanaChampaign, Urbana, IL 61801, USA. Correspondence and requests for materials should be addressed to P.S.F. (email: p.freemont@imperial.ac.uk)
} 
(c) Open Access This article is licensed under a Creative Commons Attribution 4.0 International License, which permits use, sharing, adaptation, distribution and reproduction in any medium or format, as long as you give appropriate credit to the original author(s) and the source, provide a link to the Creative Commons license, and indicate if changes were made. The images or other third party material in this article are included in the article's Creative Commons license, unless indicated otherwise in a credit line to the material. If material is not included in the article's Creative Commons license and your intended use is not permitted by statutory regulation or exceeds the permitted use, you will need to obtain permission directly from the copyright holder. To view a copy of this license, visit http://creativecommons.org/licenses/by/4.0/.

(C) The Author(s) 2019 